\title{
La Fauna Silvestre De Córdoba Y Su Rol Ecológico Aplicadas En Prácticas Socio-Comunitarias De Enseñanza
}

\section{Claudia. M. Dellafiore, $\mathrm{PhD}$}

Facultad de Ciencias Exactas, Físico-Químicas y Naturales.

Universidad Nacional de Río Cuarto, Argentina

\section{Valeria Autrán, PhD}

Facultad de Agronomía y Veterinaria.

Universidad Nacional de Río Cuarto, Argentina

\section{Delia Aiassa, PhD \\ Pablo Brandolin, PhD}

Facultad de Ciencias Exactas, Físico-Químicas y Naturales.

Universidad Nacional de Río Cuarto, Argentina

\section{Abstract}

The National Rio Cuarto University (UNRC, Cordoba, Argentina) has a scheme to encourage the inclusion of community-engagement activities (PSC) in the Higher Education curriculum. In this context, the Natural Sciences department (Faculty of Exact Sciences, Physico-Chemical and Natural) carried out a PSC activity named "Animal Biodiversity". This activity responds to specific needs by the local teaching community and the general public, where many teachers admit that they lack knowledge about the local fauna and their ecological role, and that they don't have enough time or information to develop this type of content during their scheduled teaching sessions. Therefore, the present activity aimed to liaise the Higher Education community and local primary and secondary school teachers. Biology university students taking the "Systematic Zoology" module prepared a session about "Snakes and scorpions of Cordoba. Their identification and ecological role" that included both theoretical and practical aspects for secondary school students from "Ramón Artemio Estafolani Granja Siquem" school. The analysis of the PSC activities highlighted the importance of building inclusion bridges with the local community, and the need to build social and cultural support networks. It is important to implement processes that encourage PSC in Higher Education, in order to highlight professional values, promote social responsibility and develop 
creative, active and supportive thinking. It would also provide comprehensive training of university students in ways that meet the demands and challenges of society. This work stresses the need for PSC to be continued in time by including them in our Higher Education curriculum.

Keywords: Community-engagement activities, education, wildlife animal

\section{Resumen}

En el marco de la convocatoria de la Universidad Nacional de Río Cuarto - Córdoba - Argentina a proyectos de incorporación de prácticas socio-comunitarias (PSC) a la curricula de la Educación Superior se propuso el desarrollo de la PSC denominada "Biodiversidad Animal”. Dicha PSC se desarrolló en el ámbito del Departamento de Ciencias Naturales de la Facultad de Ciencias Exactas, Físico-Químicas y Naturales. Dicha práctica se plantea a partir de una inquietud proveniente desde el ámbito educativo y de la sociedad en general. Muchos docentes reconocen que no poseen suficiente información sobre la fauna local y su rol ecológico y manifiestan que no cuentan con el tiempo ni la información suficiente para desarrollar estos contenidos en profundidad durante el desarrollo de sus asignaturas. Debido a ello, el presente trabajo tuvo por finalidad vincular en una labor conjunta a la comunidad educativa universitaria con la comunidad formativa local. Los estudiantes de la asignatura Zoología Sistemática del Profesorado en Biología prepararon una clase de capacitación, con modalidad teóricopráctica, sobre "Serpientes y escorpiones de Córdoba. Su identificación y rol ecológico" para la comunidad de la escuela media "Ramón Artemio Estafolani - Granja Siquem”. El análisis de las PSC desarrolladas ponen en evidencia la importancia de generar puentes de inclusión con el medio local y la necesidad de tender redes de apoyo social y cultural. Es importante implementar en educación superior procesos que motiven y den importancia a las PSC como disciplina, para resaltar valores profesionales, promover la responsabilidad social y desarrollar el pensamiento creativo, activo y solidario. Dar formación integral a los estudiantes universitarios de manera tal que respondan a exigencias y desafíos de la sociedad. Este trabajo destaca la necesidad de continuidad en el tiempo de las PSC afianzándolas mediante la inclusión en la currícula de nuestra casa de altos estudios.

Palabras clave: Prácticas socio-comunitarias, educación, fauna silvestre.

\section{Introducción:}

En el marco de la convocatoria de la Universidad Nacional de Río Cuarto, Córdoba, Argentina que motiva la incorporación de Prácticas SocioComunitarias (PSC) a la curricula de la Educación Superior se propuso el 
desarrollo de la PSC denominada "Biodiversidad Animal" en el marco de la carrera del Profesorado en Ciencias Biológicas de la Facultad de Ciencias Exactas, Físico-Químicas y Naturales de dicha Universidad.

La implementación de PSC surge como una preocupación existente de parte de las instituciones por demostrar un comportamiento responsable en su entorno, generando compromisos con el entorno, la sociedad, el medio ambiente (Ramírez Lozano, 2015). A su vez, la descripción de las prácticas de aplicación socio-educativa promueven y posibilitan el establecimiento de redes sociales y de comunidades más inclusivas e integradoras (JiménezRamírez, 2012). Entre los beneficios académicos para los estudiantes se enfatiza el dominio sobre el contenido de las asignaturas, habilidades de pensamiento y resolución de problemas, la realización de práctica docente y el reconocimiento y aceptación de realidades diferentes. Por lo tanto, es importante implementar en la educación superior procesos que motiven y den importancia a la prácticas socio-comunitarias como estrategia, para resaltar valores profesionales, promover una manifiesta responsabilidad social y desarrollar el pensamiento creativo, activo y solidario (Arratia Figueroa, 2008).

La relación entre la Universidad y la sociedad se entiende como de diálogo, comunicación, cooperación y aprendizaje conjunto, enriqueciendo la actividad académico-científica de la Universidad con su interacción y comunicación con diversos ámbitos de la realidad social (García, 2007). La responsabilidad social es un tema que está tomando mayor preponderancia y se discute en diferentes ámbitos (empresariales, académicos y políticos) y en las últimas décadas, se ha convertido en una referencia permanente que progresivamente está siendo aplicada por las organizaciones de todo tipo (Ramírez Lozano, 2015).

Los mayores desafíos en la formación universitaria, consiste en integrar capacidades cognitivas en los ámbitos emocional y social. El desarrollo integral de las personas debe tender al logro de competencias que respondan a exigencias y desafíos de la sociedad tanto como al proyecto inherente al individuo (Arratia Figueroa, 2008). En este marco pedagógico, los estudiantes son protagonistas de su aprendizaje y del servicio que realizan. El estudiante debe formar una personalidad de autosuficiencia, autoestima y capacidades de resiliencia y liderazgo; por lo tanto, es importante promover actitudes positivas y realistas en torno al trabajo y fortalecer valores morales y habilidades para tomar responsabilidades. Además, es deseable que adquieran destrezas para trabajar productiva y solidariamente en pos de la sociedad. Es importante que los estudiantes tengan la posibilidad de discutir acerca de las actividades a programar, identificar necesidades de la comunidad, reconocer problemas y recolectar datos, implementar y evaluar actividades para la resolución de estos 
problemas y proponer formas de presentarlos a la comunidad (Arratia Figueroa, 2008). Son ellos quienes, guiados por profesores elaboran un proyecto, lo llevan a cabo y lo evalúan aprendiendo de su práctica a través de la reflexión.

Si bien es de larga trayectoria este tipo de actividades dentro de nuestra organización educativa de estudios superiores, donde denodadamente diversos equipos de integrantes de la comunidad educativa han transitado a través de los años desde diversos proyectos de extensión, voluntariado, responsabilidad social universitaria, aprendizaje y servicio, entre otras actividades; en su Plan Estratégico Institucional se establece como lineamiento la pertinencia social y solidaria entendida como enseñanza, producción y utilización de conocimientos tecnológicos y científicos para contribuir a pensar y resolver los problemas críticos de la región así como la formación de los estudiantes (RCS N³22/09, U.N.R.C, 2009).

Por todo lo expresado, surge la necesidad de incluir en la currícula estas experiencias como modo de extensión, reconocimiento e institucionalización de las mismas de manera tal "que integre: formación general, profesional específica, científico-técnica y socio-humanística; que contribuya a la creación de conciencia social y ciudadana, en el marco de una función crítica de la Universidad” (RCS N³22/09, U.N.R.C, 2009).

Dada la imperancia del tema es que se resuelve comenzar una etapa transitiva hacia la concreción de nuevos espacios curriculares destinados a estos aprendizajes. De esta manera y concibiendo a la escuela como una comunidad, avanzamos en el análisis de la colaboración que se establece entre el centro educativo, otras organizaciones afines y los recursos del entorno, con la finalidad de tender redes sociales y alianzas que contribuyan a posibilitar un sostén para la integración socio escolar del estudiantado en situación de exclusión (Jiménez-Ramírez, 2012).

Los estudiantes universitarios a través de sus prácticas, permiten repensar el trabajo educativo en la sociedad, para la sociedad y con la sociedad (Caride Gómez, 2005). Surge de este modo, la intervención de nuestro equipo de trabajo mediante el Proyecto de Prácticas Socio Comunitarias “Biodiversidad Animal”. A partir de una inquietud proveniente del ámbito educativo, donde muchos docentes del medio reconocen que no poseen suficiente información sobre la fauna local y de su rol ecológico y que no cuentan con el tiempo ni la información suficiente para desarrollar estos temas en profundidad durante el dictado de sus asignaturas. Los objetivos del presente trabajo se plantean en función de vincular en una labor conjunta a la comunidad educativa universitaria con una comunidad formativa local en lo relacionado a la Fauna Silvestre y su rol ecológico.

Numerosas publicaciones científicas, organizaciones y gobiernos dan cuenta de la alarmante tasa de extinción de especies que la tierra está 
enfrentando en la actualidad. Si bien la extinción forma parte del curso natural de la historia del planeta este episodio de extinción es el más grande que el mundo ha experimentado en los últimos 65 millones de años (PNUMA, 2010). Esta extinción en masa, se debe en gran medida, al enorme crecimiento del área destinada a la producción agrícola ganadera. Tal es así, que el crecimiento mundial en la producción de cultivos en los últimos 50 años, se ha triplicado agricultura (FAO, 2013). Esto se debe a diversos factores entre ellos, el aumento de rendimiento por unidad de tierra, menores períodos de barbechos entre cultivos, la intensificación de la producción y la expansión de las fronteras agrícolas en detrimento de áreas de relictos naturales (Autrán et al., 2013). Existe por lo tanto, pleno consenso científico y social en referencia a la importancia de la biodiversidad para el funcionamiento y mantenimiento de los ecosistemas.

La diversidad es un elemento esencial para la estabilidad y la complejidad del agro ecosistema, aportando mayor resistencia a las perturbaciones, menor vulnerabilidad de cultivos a enfermedades y plagas y en el mismo sentido, trae beneficios tales como la prevención de la erosión del suelo (Altieri, 1999 y Winograd, 1995). La biodiversidad posee valores asociados a regulaciones en los procesos biológicos como reciclado de material orgánico y nutrientes; flujo de la energía; mecanismos de regulación biótica, que garantizan la preservación de la estructura, estabilidad y resiliencia de los sistemas (Marasas et al., 2012).

"Solo amamos aquello que conocemos", esta frase de Jack Cousteau sintetiza la importancia de que la sociedad conozca con seriedad científica nuestro patrimonio natural. Es necesario revalorizar lo que ya se conoce y ampliar el conocimiento hacia lo desconocido desde el afecto y el respeto. No hay desarrollo sustentable posible si no se conocen los recursos naturales propios de la región y su rol ecológico y no se trabaja respetando los numerosos valores intrínsecos, sociales, estéticos, culturales, tradicionales y espirituales de la biodiversidad.

La biodiversidad animal es la más sensible frente a los cambios ambientales y se ve impactada tanto por factores indirectos, como son las actividades industriales, ganaderas, agrícolas, urbanas, etc., como por factores directos como la caza ilegal, la caza de subsistencia, la captura de animales para su comercialización la matanza por conflictos con la actividad productiva o por ser considerados poco carismáticos o "peligrosos", etc. Todos los sectores de la sociedad se relacionan, desde diferentes ámbitos, con la fauna autóctona pero son las comunidades rurales y las de bajo recurso las que mantienen una relación más estrecha con la fauna silvestre. Por ejemplo, es usual la captura de ejemplares de aves para venderlos directamente a personas interesadas o a negocios locales que trafican con la fauna silvestre. Otra actividad común es la de guiar a los cazadores furtivos a 
lugares con especies de interés, como puma, venados, ñandúes, etc., que en la mayoría de los casos son especies en peligro de extinción y/o endémicas (Dellafiore, obs. pers.)

Sobre la base de todo lo mencionado se planificó entonces la articulación con la comunidad educativa "Ramón Artemio Estafolani” de la "Granja Siquem” ubicada en la zona peri-urbana de la ciudad de Río Cuarto, Córdoba, Argentina. Actualmente dicha organización cuenta con un convenio de colaboración con la Universidad Nacional de Río Cuarto. En este centro se ofrece formación y capacitación a estudiantes que viven en sectores aledaños a la ciudad en cercanía del sector agrario; que provienen de una franja social vulnerable, debido en muchos casos al entorno marginal que los rodea en su vida cotidiana.

Los objetivos de la presente PSC son:

Objetivos de la Comunidad educativa de la UNRC

- Desarrollar actitudes de solidaridad, sensibilidad y compromiso social en los estudiantes universitarios

- Dar a conocer la especies de serpientes y escorpiones de Córdoba, su rol ecológico y las medidas de precaución y prevención de accidentes

- Fomentar el aprendizaje de la comunicación y transmisión de los conocimientos relacionados con la biodiversidad animal al conjunto de la sociedad a través de su interacción con problemas de la realidad social y al servicio a la resolución de problemas sociales

- Transmitir el afecto y respeto por la diversidad animal entendiendo que no es posible concebir la existencia humana al margen de la naturaleza.

Objetivos de Trabajos con la Comunidad educativa "Ramón Artemio Estafolani:

- Adquirir conocimientos sobre la fauna regional y su rol ecológico

- Conocer los valores de la biodiversidad animal

- Adquirir conocimientos sobre especies de vertebrados e invertebrados venenosas locales y las medidas de prevención.

\section{Materiales y Métodos}

Los estudiantes de la UNRC prepararon una clase con modalidad teórico-práctica de capacitación utilizando la temática de "Serpientes y escorpiones de Córdoba y su rol ecológico" para la comunidad de la escuela "Ramón Artemio Estafolani" que funciona dentro de la "Granja Siquem". 
Dicha actividad se desarrolló en el marco de la asignatura de Zoología Sistemática del tercer año de la carrera del Profesorado en Ciencias Biológicas de la Facultad de Ciencias Exactas, Físico-Químicas y Naturales.

El espíritu participativo, de compartir y complementar conocimientos mediante la socialización de los mismos en cuanto al tema a tratar fue el eje central del encuentro. Se elaboraron materiales audiovisuales utilizando power point y material escrito en formato papel para la identificación de las especies de serpientes y escorpiones de Córdoba.

Para la elaboración de material de trabajo/divulgación se tuvieron en cuenta referencias gráficas de grado de peligrosidad, tamaño, colores y áreas de distribución de los animales. También se contemplaron las pautas de cómo actuar en caso de encontrar los distintos animales, haciendo hincapié en la peligrosidad de la manipulación de los mismos, principalmente de los venenosos aún después de muertos.

Finalmente se hicieron encuestas a directivos, docentes y estudiantes de la escuela Ramón Artemio Estafolani y a los estudiantes universitarios con el objetivo de evaluar el grado de satisfacción de los diferentes participantes frente a la capacitación y las necesidades futuras relacionadas con la biodiversidad animal.

\section{Resultados y Discusión}

Durante el desarrollo de la actividad de PSC se logró difundir y dar a conocer la fauna regional generando un lazo de afecto y respeto entre la sociedad y la biodiversidad animal, el rol ecológico y los servicios ecosistémicos de la fauna autóctona, transmitir la importancia de la diversidad animal y adquirir conocimientos sobre especies venenosas y sus medidas de prevención.

La estrategia desarrollada fue muy enriquecedora y pone en evidencia la necesidad de continuar afianzando las actividades de cooperación y aprendizaje conjunto entre la Universidad y diferentes sectores de la sociedad. Así, según las necesidades de los entornos de los centros educativos y la función desarrollada desde la universidad, con una perspectiva sistémica y ecológica, se posibilita un nexo entre la dimensión social de diversos ámbitos de la realidad para fomentar la construcción del conocimiento fundamentándose en las comunidades de aprendizaje. Tan es así, que esta premisa sirvió para analizar los resultados por franjas relacionadas a los roles de los diversos actores de la comunidad educativa que han permitido la concreción del trabajo (directivos, docentes, estudiantes universitarios y del centro educacional "Ramón Artemio Estafolani”);

En este sentido se permitió el desarrollo de actitudes de solidaridad, sensibilidad y compromiso social en cada una de las esferas. El encuentro fue dinámico y participativo, enriquecedor en todos los sentidos, cuenta de 
ello se hace referencia a las respuestas dadas en las encuestas (Figuras 1, 2 y 3).

Se intercambiaron experiencias, reflexionando sobre el importante rol ecológico y la importancia en el medio, como actuar para prevenir accidentes y que hacer en casos de mordeduras o picaduras. La importancia de la higiene cerca de los hogares y las acciones que deben evitarse para que se generen refugios de animales indeseables en ese marco familiar. Se puso énfasis en especies en peligro de extinción y otras como controladores biológicos de plagas rurales y peri-urbanas referenciando la importancia de mantenerlas con vida y en su hábitat natural (y no como mascotas) ya que no afectan la vida del hombre, sino que colaboran en ella.

La evaluación de lo actuado fue analizada en primer lugar en relación con los directivos y docentes de la organización donde se brindó la PSC a los cuales les resultó interesante el tema de la charla (100\% de los encuestados) dado que la temática de la práctica versó sobre animales que los integrantes de la comunidad educativa suelen ver y que los niños y jóvenes, desconociendo su peligrosidad, las toman y utilizan para jugar o molestar a compañeros, como mascotas e incluso las matan desconociendo su rol ecológico.

Los encuestados (directivos, docentes y alumnos del centro educacional "Ramón Artemio Estafolani”) consideran que los estudiantes universitarios fueron claros en los conceptos dados y sus explicaciones y que emplearon un lenguaje sencillo. En cuanto al material audiovisual de apoyo usado opinan que ayudó al desarrollo de la clase. Fueron respondidas todas las preguntas generadas durante el encuentro por lo que se propició una interacción positiva entre los participantes. Refieren que la clase fue dinámica y permitió poner en relevancia los conocimientos previos de los estudiantes. Por todo lo anterior, expresan que les gustaría repetir este tipo de actividades en el futuro y que las mismas sean constantes en el tiempo. Sugieren otros temas relacionados con la fauna silvestre que les gustaría les fuera brindado como por ejemplo: animales en peligro de extinción, importancia de su conservación, rol de la fauna silvestre en el medio rural, aves, liebres, etc.

Las encuestas a los estudiantes de la Universidad que fueron los encargados de llevar adelante el encuentro indican que fue muy valiosa la experiencia ya que fue la primera vez en toda la carrera que están en una situación real propia del alcance de su futuro título. Además, manifestaron que fueron muy valiosos los aportes de los docentes de la asignatura de Zoología Sistemática en relación a los consejos de cómo organizar una clase atractiva, participativa y didáctica y la importancia de valorizar la participación de los estudiantes en la misma (Figura 1). Respondieron que es importante tener experiencias con otros niveles de la educación para motivar 
a los futuros profesores y opinan que debe repetirse con los compañeros que cursen la asignatura los próximos años.

\section{¿Le resultó valiosa la experiencia?}

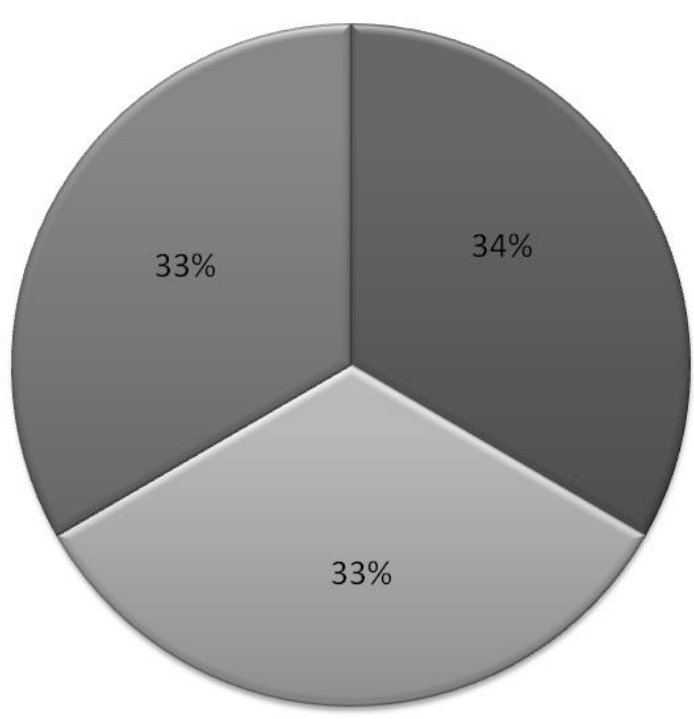

Sí, fue la primera experiencia y nos ayudó a realizar una práctica docente frente al aula. En la currícula del profesorado solo realizamos prácticas en el último año de la carrera.

$\square$ Fue genial!! (Fue muy Buena) Nunca me había tocado ingresar a una escuela a dar una charla. Fue muy emocionante

Fue muy valiosa, nos sirvieron mucho los consejos de los docentes de cómo organizar una clase entretenida y fue muy importante ver la participación de los chicos en la misma.

Figura 1. Encuesta a estudiantes del Profesorado en Ciencias Biológicas de la Universidad Nacional de Río Cuarto relacionada a la PSC.

\section{¿Le resulto interesante el tema de la charla? Justifique su respuesta.}

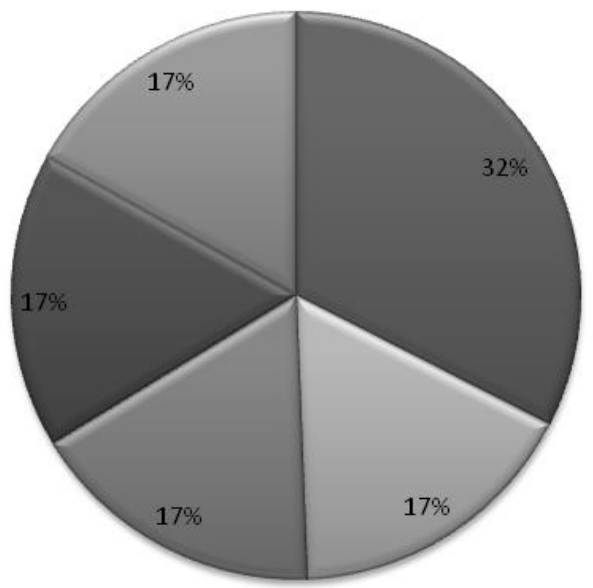

- Me pareció importante la charla porque ayuda a prevenir accidentes y te avisa que hay que tener cuidado

$\square$ Aprendí las características de todos los animales

- Si es muy importante por las dudas que se me aparezcan víboras o alacranes se cómo distinguir si es venenoso o no

Me pareció importante porque no conocía algunas serpientes

Es interesante aprender las características de los animales venenosos y no venenosos.

Figura 2. Encuesta a estudiantes de escuela "Ramón Artemio Estafolani -Granja Siquem" en relación a la temática de la clase de capacitación. 
¿Le gustó la presentación? ¿Le gustaron los materiales usados durante la charla?. Nos gustaría nos justifique la respuesta.

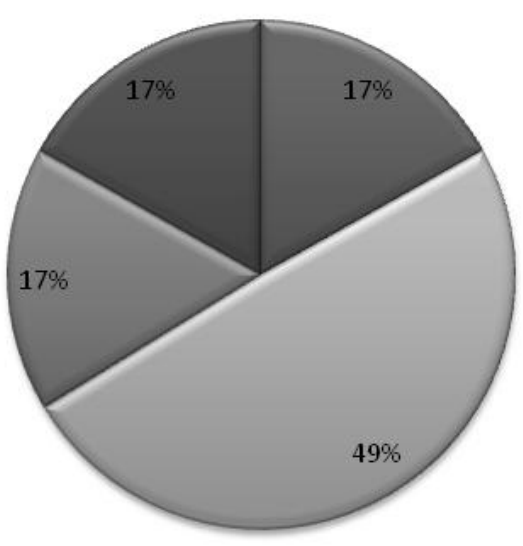

Si me gustó aprender sobre serpientes y escorpiones

Si muchísimo, me gusto aprender las diferencias de las serpientes. Algunas no las conocía

Me gustaron las imágenes

Me gustó el tema de los escorpiones y sus diferencias

Figura 3. Encuesta a estudiantes de escuela "Ramón Artemio Estafolani - Granja Siquem" en relación a los materiales usados durante la clase de capacitación.

\section{Conclusión}

La PSC desarrollada por docentes y estudiantes de la UNRC resultó ser una experiencia muy valiosa para establecer lazos con el medio. Surge la importancia de que dichos lazos se mantengan en el tiempo ya que se puso en evidencia la necesidad de repetir dicha actividad en el futuro e incluir nuevas prácticas con los temas propuestos por la comunidad educativa que fue la receptora de esta experiencia (Escuela Ramón Artemio Estafolani Granja Siquem).

En el marco de las PSC, los estudiantes son protagonistas de su propio aprendizaje y del servicio que realizan. Al aprender y preparar los contenidos a enseñar los estudiantes, influenciados por sus ideas actuales, ayuda a su aprendizaje. Por lo tanto, es útil pensar que los resultados de este tipo de aprendizaje es un proceso de cambio conceptual, incluyendo tanto las extensiones de conocimiento como el intercambio de concepciones (Agiande et al., 2015).

A nivel global, la responsabilidad social es un nuevo paradigma organizacional que está integrado voluntariamente, sin ninguna imposición legal, en las estrategias de las empresas (Ramírez Lozano, 2015). Respondiendo a esta tendencia, es importante implementar en educación superior procesos de responsabilidad social que motiven y den importancia a las PSC mediante su inclusión en la currícula de grado. Desde la mirada de los docentes de la asignatura donde se enmarcó la estrategia surge que los 
estudiantes universitarios lograron implicarse profundamente en la actividad y demostraron una gran satisfacción al participar de dicha experiencia.

\section{Agradecimientos}

Agradecemos a la Secretaría de Planeamiento y Relaciones Institucionales de la Universidad Nacional de Río Cuarto por al apoyo financiero, a las autoridades y alumnos de la Granja Siquem y a los alumnos Rosa Arabel, Florencia Lescano y José Torres.

\section{References:}

1. Agiande, D.U., Williams, J.J., Dunnamah, A.Y., Tumba, D.P. 2015. Conceptual change theory as a teaching strategy in environmental education. European Scientific Journal 11(35).

2. Altieri, M.A. 1999. Agroecología: bases científicas para una agricultura sustentable. Nordan Comunidad. Montevideo. Uruguay. 70 pp.

3. Arratia Figueroa, A 2008. Ética, solidaridad y "aprendizaje servicio" en la educación superior. Acta bioethica, 14(1), 61-67. http://www.scielo.cl/scielo.php?pid=S1726569X2008000100008\&script=sci_arttext\&tlng=e

4. Autrán, V.A, Puricelli, E.C, \& Andrés, J.A. 2013. Fitotoxicidad de herbicidas postemergentes sobre Adesmia bicolor (Poir.) DC y control de malezas asociadas. Agriscientia, 30(2), 57-67. http://www.scielo.org.ar/scielo.php?script=sci_arttext\&pid=S1668298X2013000200002\&lng=es\&tlng=es.

5. Caride Gómez J. A. 2005. La animación sociocultural y el desarrollo comunitario como educación social. Revista de Educación, núm. 336, pp. 73-88 *http://www.revistaeducacion.mec.es/re336/re336_05.pdf

6. FAO. 2013. Statistical Yearbook 2013. Agricultura y la alimentación. Organización de las Naciones Unidas. Roma junio 2013. Disponible en: http://www.fao.org/economic/ess/ess-publications/essyearbook/en/. Acceso el 2 de octubre de 2016.

7. García M R. 2007. Interacción y comunicación en entornos educativos: Reflexiones teóricas, conceptuales y metodológicas”. Revista da Associação Nacional dos. Programas de Pós-Graduação em Comunicação Interacción.

8. Jiménez-Ramírez, M. 2012. Actuaciones socio-comunitarias y educativas inclusivas con alumnado en riesgo de exclusión social. Revista de Investigación en Educación. ISSN: 1697-5200 / eISSN 2172-

3427http://reined.webs.uvigo.es/ojs/index.php/reined/article/viewFile /494/233 
9. Marasas, M.; G. Cap.; L. De Luca; M. Pérez y R. Pérez. 2012. El camino de la transición agroecológica. INTA. Mariana Edith. Ciudad Autónoma de Buenos Aires: Ediciones INTA. (1): 90 pp.

10. PNUMA, 2010 http://www.unep.org/spanish/wed/2010/biodiversity.asp

11. Ramírez Lozano, J.P. 2015. Creación de reputación corporativa mediante la adecuada gestión de la responsabilidad social. Estudio del caso de las empresas: BCP, Kimberly Clark y AFP Integra-Sura. European Scientific Journal 11(14).

12. U.N.R.C. 2009. Resolución Consejo Superior N 322/09 Creación PSC.U.N.R.C.

13. Winograd, M. 1995. Indicadores ambientales para Latinoamérica y el Caribe: hacia la sustentabilidad en el uso de tierras, IICA, San José de Costa Rica. 84 p.

\section{ANEXO 1 \\ ENCUESTA ALUMNOS COMUNIDAD EDUCATIVA PRÁCTICAS SOCIOCOMUNITARIAS (PSC)}

Nombre y Apellido:

Escuela/Colegio:

Curso:

1. ¿Le resulto interesante el tema de la charla?. Justifique su respuesta.

2. ¿Le resulto entendible la charla?. ¿Puede explicarnos porque si o porque no?

3. ¿Le gustó la presentación? ¿Le gustaron los materiales usados durante la charla?. Nos gustaría nos justifique la respuesta.

4. ¿Le gustaría conocer algún tema en especial en relación con los animales silvestres? ¿Cuál?.

5. Por favor describa SUGERENCIAS que considere puedan mejorar esta actividad.

MUCHAS GRACIAS POR TU PARTICIPACIÓN!!!! TU OPINIÓN ES MUY IMPORTANTE PARA NOSOTROS!!! 


\section{ANEXO 2}

\section{ENCUESTA ALUMNOS UNRC PRÁCTICAS SOCIOCOMUNITARIAS (PSC)}

Nombre y Apellido:

Fecha realización de la práctica:

Tema seleccionado para la práctica:

Te solicitamos contestes este breve cuestionario con el objetivo de conocer tu opinión sobre las Prácticas Socio-comunitarias. Este tipo de prácticas son el resultado de un importante esfuerzo por parte de los docentes y de la Universidad Nacional quien financia este tipo de actividades. Como futuro profesional en Ciencias Biológicas nos interesa saber que te ha parecido esta actividad... “tu opinión es muy importante para nosotros”. Desde ya Muchas Gracias!!!

1. ¿Se le explicaron correctamente los objetivos y alcance de la PSC?

a. SI / NO

b. En caso negativo justifique su respuesta.

2. ¿Le pareció adecuado el tema seleccionado para la charla/clase que se dictó a la comunidad educativa?

a. SI / NO

b. ¿por qué?.

3. ¿Tuvo tiempo suficiente para la preparación de la charla/clase?

a. SI / NO

b. En caso de ser negativo justifique

4. ¿Le pareció adecuado el tiempo usado para la charla/clase?

a. SI / NO

b. En caso de ser negativo justifique

5. ¿Las herramientas que se le brindaron para el dictado de la charla/clase fue el adecuado?. SI / NO ¿por qué?.

6. ¿Le resulto valiosa la experiencia? Justifique.

7. ¿Cree que este tipo de experiencia debe repetirse con los compañeros que cursen la asignatura los próximos años? SI / NO ¿por qué?.

8. ¿Le gustaría realizar otras prácticas de este tipo en otras asignaturas?. En caso de que su respuesta sea afirmativa puede sugerir alguna asignatura en la que le gustaría contar con PSC.

9. Por favor describa sugerencias que considere puedan mejorar esta actividad. 


\section{ANEXO 3}

\section{ENCUESTA AUTORIDADES Y DOCENTES CITER PRÁCTICAS SOCIOCOMUNITARIAS (PSC)}

Nombre y Apellido:

Fecha realización de la práctica:

Nos gustaría conocer tu opinión sobre las Prácticas Socio-comunitarias. Este tipo de prácticas son el resultado de un importante esfuerzo por parte de los docentes y de la Universidad Nacional de Río Cuarto. Es nuestra intención mejorar y avanzar en el desarrollo de este tipo de actividades.

Desde ya muchas gracias!!!

1. ¿Le resulto interesante el tema de la charla?. Nos gustaría nos justifique la respuesta.

2. ¿Le resulto entendible la charla?. ¿Puede explicarnos porque si o porque no?

3. ¿Le gustó la presentación? ¿Le gustaron los materiales usados durante la charla?. Nos gustaría nos justifique la respuesta.

4. ¿Le gustaría repetir este tipo de actividades en el futuro?

5. ¿Hay algún otro tema relacionado con la fauna silvestre que le gustaría le fuera brindado a los alumnos? ¿Cuál?.

6. Por favor describa SUGERENCIAS que considere puedan mejorar esta actividad.

\section{MUCHAS GRACIAS POR LA ATENCIÓN BRINDADA Y POR COLABORAR CON ESTA ENCUESTA!!!}

